\title{
Long non-coding RNA profiles in plasma exosomes of patients with gastric high-grade intraepithelial neoplasia
}

\author{
FENG HU $^{1}$, MIN RAO ${ }^{1}$, MANLI ZHANG ${ }^{1}$, QINGQING MENG ${ }^{1}$, MINJIE WAN $^{2}$, \\ XIUNA ZHANG ${ }^{1}$, LILI DING ${ }^{3}$ and YANFANG JIANG ${ }^{4}$ \\ ${ }^{1}$ Department of Hepatology and Gastroenterology, The Second Part of First Hospital of Jilin University; Departments of \\ ${ }^{2}$ Central Laboratory and ${ }^{3}$ Intensive Care Unit; ${ }^{4}$ Key Laboratory of Organ Regeneration and Transplantation of The \\ Ministry of Education, Genetic Diagnosis Center, The First Hospital of Jilin University, Changchun, Jilin 130000, P.R. China
}

Received October 15, 2020; Accepted September 22, 2021

DOI: $10.3892 /$ etm.2021.10923

\begin{abstract}
Long non-coding (lnc) RNAs in circulating exosomes are a new class of promising cancer biomarkers; however, their expression in exosomes derived from gastric high-grade intraepithelial neoplasia (GHGIN) has not been reported. In the present study, differentially expressed (DE) lncRNAs were analyzed in the peripheral blood collected from 5 patients with GHGIN and 5 healthy donors using high-throughput sequencing. Reverse transcription-quantitative PCR analysis was performed on 6 randomly selected DE lncRNAs to validate the reliability of the sequencing results. The potential roles of the DE IncRNAs in GHGIN were investigated using Gene Ontology (GO) and Kyoto Encyclopedia of Gene and Genome (KEGG) pathway enrichment analyses. A total of 25,145 lncRNAs were identified in all the samples and 83 DE lncRNAs were further screened, including 76 upregulated and 7 downregulated DE 1ncRNAs. GO and KEGG analyses predicted that the DE IncRNAs played notable roles in 'protein/macromolecule glycosylation', 'regulation of protein ubiquitination', 'renin-angiotensin system' and 'MAPK signaling pathways'. A lncRNA-micro (mi)RNA-mRNA interaction network was constructed and used to perform association analyses. It was found that 83 lncRNAs were abnormally expressed in GHGIN, with some potential functions associated with gastric cancer. Furthermore, the lncRNA-miRNA-mRNA interaction network indicated that 7 DE IncRNAs may play a notable role in the occurrence and development of GHGIN. The results of the present study showed the expression profiles of IncRNAs in human GHGIN, elucidated some of the molecular changes associated with
\end{abstract}

Correspondence to: Professor Yanfang Jiang, Key Laboratory of Organ Regeneration and Transplantation of The Ministry of Education, Genetic Diagnosis Center, The First Hospital of Jilin University, 3302 Jilin Road, Changchun, Jilin 130000, P.R. China E-mail: yanfangjiang@jlu.edu.cn

Key words: high-grade intraepithelial neoplasia, exosomes, long non-coding RNAs, high-throughput sequencing, gastric cancer
GHGIN and improved the understanding of the molecular mechanisms underlying GHGIN and gastric cancer.

\section{Introduction}

Gastric cancer (GC), one of the most common fatal types of cancer, was the 5th most regularly diagnosed tumor and the 3rd most common cause of tumor-related death worldwide in 2018 (1). Advances have been made in the diagnosis and treatment of GC; however, the prognosis of patients with GC remains poor, with $>70 \%$ of patients eventually succumbing to the disease (2). Therefore, early diagnosis and treatment is crucial for improving prognosis (3). GC is the final result of long-term biological processes, including the gradual accumulation of genotypical and phenotypical changes (4). Precancerous lesions, pathologically defined as dysplasia, have been associated with the development of GC. Dysplasia can be further classified as low- or high-grade intraepithelial neoplasia (HGIN) (5). HGIN is equivalent to severe allogeneic hyperplasia and carcinoma in situ (4). A previous study reported that $59.1 \%$ of patients with gastric high-grade intraepithelial neoplasia (GHGIN) progress to gastric adenocarcinoma within one year after endoscopy (6). Thus, early detection of precancerous lesions, particularly GHGIN, is important for the prevention of GC and reducing its associated mortality rate. Currently, endoscopy followed by pathological examination is the most reliable diagnostic tool for GHGIN; however, it is invasive and uncomfortable. Therefore, non-invasive, cost-effective and highly sensitive biomarkers are needed to diagnose GHGIN.

Some studies have explored the role of exosomes in local and systemic intercellular communication during cancer progression $(7,8)$, and some studies suggest the potential application of exosomes in cancer screening and diagnosis $(9,10)$. Exosomes are small extracellular vesicles (30-100 $\mathrm{nm}$ in diameter), within a lipid bilayer membrane that encompasses cytoplasm without organelles, and are highly heterogeneous, possibly reflecting the phenotypic state of the cells that produced them (11). Exosomes can transfer bioactive substances, such as proteins, coding RNAs, non-coding (nc)RNAs and DNA from donor to recipient cells, resulting in the differentiation of genetic and epigenetic factors and the 
reprogramming of target cells $(11,12)$. Some exosomes from cancer cells, such as glioma, gastric cancer and breast cancer reportedly promote angiogenesis, regulate the immune system and remodel the microenvironment, all of which are factors that facilitate cancer progression $(13,14)$. In addition, exosomes are present in almost all body fluids, which reinforces their potential application as non-invasive biomarkers for the diagnosis of various types of cancer (15).

Long non-coding (lnc)RNAs are transcripts $>200$ nucleotides in length, that have no or limited protein-coding capacity; however, they play a crucial role in genetic regulation at the epigenetic, transcriptional and post-transcriptional levels (16). In addition to mutations or abnormal expression levels of protein-coding genes, mutations and mis-regulation of ncRNAs, particularly lncRNAs, appear to play noteworthy roles in cancer, such as prostate cancer, stomach cancer and lung cancer (17). Increasing evidence indicated that IncRNAs were associated with the initiation and development of tumors, such as non-small cell lung cancer and gastric cancer, directly or indirectly via gene expression $(18,19)$. Furthermore, IncRNAs are selectively distributed to exosomes and used as signal messengers for intercellular communication (20). Thus, exosomal lncRNAs can reshape the tumor microenvironment, thereby promoting tumor development, metastasis, angiogenesis and chemoresistance (15). In addition, IncRNAs in exosomes are not affected by ribonuclease-mediated degradation and are stable in body fluids (21). Abnormal lncRNAs have been found in different types of tumors, suggesting their potential as molecular markers (22-24). The serum level of LINC00310 is increased in patients with breast cancer compared with healthy controls, with a sensitivity and specificity of 77.08 and $87.23 \%$, respectively. These levels are valuable for breast cancer diagnosis (25). Colorectal cancer-associated HOTAIR also exhibited oncogenic properties. High expression levels of HOTAIR in tumour tissues was closely associated with vascular invasion and metastasis. Patients with high expression levels of HOTAIR were more likely to have a poor prognosis (26). Therefore, IncRNAs have attracted increasing attention in the field of tumor-derived exosome research.

Some studies have revealed that plasma exosome lncRNAs are potential biomarkers for GC $(23,24)$. However, the expression profile of lncRNAs in circulating exosomes in patients with GHGIN remains to be elucidated. Therefore, the current study aimed to discover new lncRNA biomarkers for GHGIN, and investigate the carcinogenic mechanism of GHGIN and GC. The expression profiles of circulating exosomal lncRNAs in patients with GHGIN and healthy controls were compared using high-throughput sequencing to identify differentially expressed (DE) lncRNAs. In addition, the potential roles of lncRNAs and their predicted targets were elucidated by constructing a lncRNA-micro(mi)RNA-mRNA interaction network. The study findings suggested sensitive and specific non-invasive diagnostic markers for GHGIN.

\section{Materials and methods}

Patients and sample collection. In the present study, 5 patients diagnosed with GHGIN and free of any other type of cancer were recruited at The First Hospital of Jilin University from
February to June 2019. The inclusion and exclusion criteria was as follows: i) All enrolled patients were pathologically confirmed as GHGIN, and all pathological diagnoses were reviewed by pathologists in our hospital; ii) Patients exhibited no history of other tumors or other serious underlying diseases; iii) Patients did not receive any preoperative treatment; and iv) All samples were collected with informed consent of patients and healthy volunteers, and approved by the Ethics Committee of the First Hospital of Jilin University. All the patients underwent endoscopic submucosal dissection for treatment. All specimens were diagnosed as GHGIN by two independent professional pathologists according to the World Health Organization Classification of Tumors of the Digestive System (27). A total of 5 healthy donors, without a history of precancerous lesions or cancer, were included in the control group, the healthy control (HC). Table I and Fig. S1 shows the clinical features and the pathological examination images of the study participants, respectively. Subsequently, $\sim 10 \mathrm{ml}$ of peripheral blood was collected in an EDTA anticoagulation tube. The plasma was immediately separated by centrifugation $(1,900 \mathrm{x} \mathrm{g} ; 10 \mathrm{~min})$ at $4^{\circ} \mathrm{C}$, then the supernatant (plasma) was collected and centrifuged again $(3,000 \mathrm{x} \mathrm{g}$; $15 \mathrm{~min}$ ) at $4^{\circ} \mathrm{C}$ and stored as soon as possible at $-80^{\circ} \mathrm{C}$ until use. The study protocol was approved by The Ethics Committee of the First Hospital of Jilin University, and all the procedures were performed in accordance with The Declaration of Helsinki. All participants provided written informed consent.

Exosome isolation. The exosomes were isolated from the plasma samples using an exoEasy Maxi kit (Qiagen $\mathrm{GmbH}$ ) according to the manufacturer's instructions and stored at $-80^{\circ} \mathrm{C}$ until use. Briefly, buffer XBP was mixed with the plasma from the patients, then the plasma/XBP mixture was added into the exoEasy spin column and centrifuged at $500 \mathrm{xg}$ for $1 \mathrm{~min}$ at $4^{\circ} \mathrm{C}$. Buffer XWP was added to remove the residual buffer from the column. The flow-through was discarded and transferred to the spin column in a fresh collection tube, then $400 \mu \mathrm{l}$ Buffer XE was added to the membrane and centrifuged at $500 \mathrm{x} \mathrm{g}$ for $5 \mathrm{~min}$ at $4^{\circ} \mathrm{C}$ to collect the exosome.

The exosome suspension was diluted to $0.5 \mathrm{mg} / \mathrm{ml}$ in PBS (28). Then, it was loaded onto a carbon-coated formvar grid and stained with $2 \%$ osmic acid. After incubation for $10 \mathrm{~min}$ at room temperature, excess fluid was blotted with filter paper and adsorbed exosomes were negatively stained with $1 \%$ phosphotungstic acid for $5 \mathrm{~min}$ at room temperature. Finally, the air-dried exosome-containing grids were observed using a HT7700 transmission electron microscope (TEM; Hitachi, Ltd.).

The particle size distribution of exosomes was detected using a nanoparticle tracking analyser (NanoFCM Profession V1.0; Xiamen Fuliu Biotechnology Co., Ltd.).

Exosome identification. Specific exosomal markers [CD9 and tumor susceptibility gene 101 (TSG101)] were detected using western blot analysis. First, the exosomes were isolated from plasma samples using an exoEasy Maxi kit (Qiagen $\mathrm{GmbH}$ ). The exosomes were lysed in standard RIPA buffer (Beyotime Institute of Biotechnology) supplemented with protease and phosphatase inhibitors (Beyotime Institute of Biotechnology) and oscillated several times. After the exosomes were fully 
Table I. Clinical features of the study participants.

\begin{tabular}{lllll}
\hline Sample group & Sample ID & Age & Sex & Biopsy site \\
\hline GHGIN & GHGIN 1 & 59 & Male & Antrum of stomach \\
& GHGIN 2 & 68 & Female & Antrum of stomach \\
& GHGIN 3 & 66 & Female & Angle of stomach \\
& GHGIN 4 & 62 & Female & Antrum of stomach \\
& GHGIN 5 & 69 & Male & Body of stomach \\
HC & HC1 & 35 & Male & Antrum of stomach \\
& HC2 & 27 & Male & Antrum of stomach \\
& HC3 & 32 & Male & Antrum of stomach \\
& HC4 & 28 & Female & Body of stomach \\
& HC5 & 30 & Antrum of stomach
\end{tabular}

GHGIN, gastric high-grade intraepithelial neoplasia; HC, healthy control.

lysed, they were centrifuged at $13,800 \times \mathrm{g}$ for $5 \mathrm{~min}$ at $4^{\circ} \mathrm{C}$ and the supernatant was collected. A BCA protein assay kit (Beyotime Institute of Biotechnology) was used to measure the protein concentration. A total of $30 \mu \mathrm{g}$ protein per lane were separated on $10 \%$ acrylamide/bisacrylamide gels and transferred to PVDF membranes. Membranes were subsequently blocked with $5 \%$ skimmed milk at $4^{\circ} \mathrm{C}$ overnight and incubated with primary antibodies at $4^{\circ} \mathrm{C}$ overnight. Following primary incubation, membranes were incubated with secondary antibodies at room temperature for $1 \mathrm{~h}$. The following antibodies were used: Rabbit anti-human CD9 (1:1,000; cat. no. 98327S; Cell Signaling Technology, Inc.), rabbit anti-human TSG101 (1:1,000; cat. no. ab125011; Abcam) and rabbit anti-calnexin (1:1,000; cat. no. 2679S; Cell Signaling Technology, Inc.). Calnexin, an endoplasmic reticulum marker, was used as the negative control. Proteins were visualized using ChemiSignal $^{\mathrm{TM}}$ ECL Plus Chemical Luminescence agent (cat. no. 1810212Shanghai Qinxiang Technology Co., Ltd.) using the Tanon 6100 chemiluminescent imaging system (Tanon Science \& Technology Co., Ltd.), according to the manufacturer's instructions.

RNA isolation and quality control. Total RNA from the exosomes was extracted using TRIzol ${ }^{\circledR}$ (Thermo Fisher Scientific, Inc.) according to the manufacturer's instructions. Briefly, $1 \mathrm{ml} \mathrm{TRIzol}{ }^{\circledR}$ was added to the exosomes collected from $1 \mathrm{ml}$ plasma, then mixed well and left to incubate for $5 \mathrm{~min}$ at room temperature. A total of $0.2 \mathrm{ml} 99.8 \%$ chloroform (Sigma-Aldrich; Merck KGaA) was added, and the samples were oscillated for $15 \mathrm{sec}$, incubated at $15-30^{\circ} \mathrm{C}$ for 2-3 min and centrifuged at $12,000 \mathrm{x}$ for $15 \mathrm{~min}$ at $4^{\circ} \mathrm{C}$. The upper aqueous phase was transferred to a new centrifuge tube and $0.5 \mathrm{ml} \mathrm{99.5 \%} \mathrm{isopropanol} \mathrm{(Sigma-Aldrich;} \mathrm{Merck} \mathrm{KGaA)}$ was added. After being oscillated, incubated at $15-30^{\circ} \mathrm{C}$ for $10 \mathrm{~min}$ and centrifuged at $12,000 \mathrm{x} \mathrm{g}$ at $4^{\circ} \mathrm{C}$ for $10 \mathrm{~min}$, the supernatant was removed. Subsequently, $1 \mathrm{ml} \mathrm{75 \%} \mathrm{ethanol}$ was added to the centrifuge tube to wash the RNA pellet; after shaking, the tube was centrifuged at 7,500 $\mathrm{x}$ g for $5 \mathrm{~min}$ at $4^{\circ} \mathrm{C}$. The ethanol solution was removed and the RNA pellet dried in the air for 5-10 min, then RNase-free water was added, mixed with the RNA pellet by pipetting several times and incubated at $55-60^{\circ} \mathrm{C}$ for $10 \mathrm{~min}$. The RNA solution obtained was stored at $-80^{\circ} \mathrm{C}$. Qualitive and quantitative analyses of the RNA samples were performed using a NanoDrop ND 1000 spectrophotometer (Thermo Fisher Scientific, Inc.) at a wavelength of $260 \mathrm{~nm}$. Denaturing agarose gel (1\%) electrophoresis was used to measure RNA integrity and genomic DNA (gDNA) contamination. When the gDNA was completely removed, the RNA integrity number was $\geq 7$ and the $\mathrm{OD}_{260} / \mathrm{OD}_{280}$ ratio was 1.8-2.1. RNA purity was thus verified and the samples were used for further experiments.

RNA library construction, and lncRNA and $m R N A$ sequencing. High-throughput RNA sequencing of all the samples was conducted by Cloud-Seq Biotech, Co., Ltd. First, rRNAs were eliminated from total RNA using the NEBNext ${ }^{\circledR}$ rRNA depletion kit (cat. no. E6350S New England Biolabs, Inc.). Then, the NEBNext ${ }^{\circledR}$ Ultra $^{\mathrm{TM}}$ II Directional RNA Library Prep kit (cat.no.E7760; New England Biolabs,Inc.) was used to construct RNA libraries from the rRNA-depleted RNAs according to the manufacturer's instructions. The BioAnalyzer 2100 system (Agilent Technologies, Inc.) was used to evaluate the quality and quantity of the libraries. $10 \mathrm{pM}$ libraries were denatured as single-stranded DNA molecules, captured on Illumina flow cells, amplified in situ as clusters and finally sequenced for 150 cycles on Illumina HiSeq Sequencer (Illumina, Inc.) according to the manufacturer's instructions.

lncRNA sequencing analysis. Cutadapt software v1.9.3 (http://code.google.com/p/cutadapt/) was used to remove connectors and low-quality reads to obtain high-quality clean reads. The results were sequenced and compared with the reference genome (UCSC hg19) using HISAT2 software v2.04 (http://www.ccb.jhu.edu/software/hisat/). Differential expression profiles of the lncRNAs and mRNAs were obtained using Cuffdiff software v2.2.1, which is part of the Cufflinks package (http://cufflinks.cbcb.umd.edu/). Fold-change (FC) and P-values were calculated based on fragments per kilobase million mapped reads to screen DE lncRNAs and mRNAs. lncRNAs with $\operatorname{logFC} \geq 2.0$ and $\mathrm{P} \leq 0.05$ in at least one sample were selected for target gene prediction. Gene Ontology (GO; www.geneontology.org) functional enrichment analysis (29) 
Table II. Randomly selected lncRNAs for reverse transcription-quantitative PCR and the primers used.

\begin{tabular}{lll}
\hline IncRNA ID & Primer direction & Primer sequence, 5'-3' \\
\hline ENST00000608199 & Forward & CGTTCTGGACCAAGCCTAAA \\
ENST00000442783 & Reverse & AGGAATTGGGAGGAGTGCTT \\
& Forward & TGGGGACAAAATGAGAGTCC \\
ENST00000427153 & Reverse & TTACAGGTGTGAGCCACTGC \\
ENST00000608625 & Forward & GAGATGCAAAGCCAGGCTAC \\
& Reverse & TCTCTGCAGCTGATTCTGGA \\
ENST00000518266 & Forward & CATCTCCCAGACCTGCTTGT \\
ENST00000523150 & Reverse & TCCCCAGCTCCCTTCTTATT \\
GAPDH & Forward & TTTGGTCATGTTTCTTGTGGT \\
& Reverse & TGGACACGCTGTCAAATTGT \\
& Forward & CAGCATATGGCCTTTGGACT \\
& Reverse & TGAGGAGACCGCAGTAAACC \\
& Forward & GGCCTCCAAGGAGTAAGACC \\
& Reverse & AGGGGAGATTCAGTGTGGTG
\end{tabular}

lncRNA, long non-coding RNA.

and Kyoto Encyclopedia of Genes and Genomes (KEGG; www.genome.jp/kegg) pathway enrichment analyses (30) of the candidate genes were conducted using the Database for Annotation, Visualization and Integrated Discovery (http://david.abcc.ncifcrf.gov). The top 10 enriched pathways in the up- and downregulated lncRNAs and mRNAs, according to the P-value cut-offs, were constructed using OmicShare tools (www.omicsshare.com/toos/).

Reverse transcription-quantitative $R T-q(P C R)$. Total RNA was isolated from exosomes using RNA extraction buffer (Sigma-Aldrich; Merck KGaA), gDNA was removed and cDNA was synthesized to form gDNA-free total RNA using the Hifair ${ }^{\circledR}$ II 1st Strand cDNA Synthesis kit (Shanghai Yeasen Biotechnology Co., Ltd.), following the manufacturer's specifications. GAPDH was used as the internal control. The primers for the 6 randomly selected lncRNAs for RT-qPCR are shown in Table II. RT-qPCR was performed by Cloud-Seq Biotech, Co., Ltd. using SYBR ${ }^{\circledR}$ Green Master Mix (Thermo Fisher Scientific, Inc.) with the QuantStudio 5 Real-Time PCR System (Thermo Fisher Scientific, Inc.), following the manufacturer's instructions. The program was setup as follows: A total of 40 cycles at $95^{\circ} \mathrm{C}$ for $10 \mathrm{sec}$ and $60^{\circ} \mathrm{C}$ for $1 \mathrm{~min}$. lncRNA expression levels were normalized to that of GAPDH and calculated using the $2^{-\Delta \Delta C q}$ method (31). Samples were analyzed in triplicate for each lncRNA.

Prediction of IncRNA-miRNA-mRNA interactions. According to the competing endogenous (ce) RNA theory (32), lncRNAs and mRNAs that demonstrated significantly different expression were selected $(\mathrm{P}<0.05)$. Subsequently, based on target lncRNA-miRNA and mRNA-miRNA information from the MiRcode database v11 (www.mircode.org), in addition to common target miRNA information (33), a lncRNA-miRNA-mRNA interaction network was constructed. To further increase the credibility of the network, only
lncRNA-miRNA-mRNA gene pairs with positive correlations between IncRNA and mRNA were retained (34). The interaction network was visualized using Cytoscape v3.8.0 software (35). Using PubMed, mRNA in the interactive network was searched to determine any potential associations with gastric cancer.

Statistical analysis. Statistical analysis was conducted using GraphPad Prism software v5.0 (GraphPad Software, Inc.). The data are expressed as the mean \pm standard deviation (unless otherwise shown). Unpaired Student's t-test was used to assess differences between two groups from three independent experiments. $\mathrm{P}<0.05$ was considered to indicate a statistically significant difference.

\section{Results}

Identification of isolated exosomes. TEM revealed the morphology and size of the extracted exosomes; they had a cup-shape appearance with a clearly defined and relatively intact membrane, ranging between 30-100 nm in size (Fig. 1A and B). The size of the exosomes was similar to the results obtained using the grain diameter distribution map. The exosome components, CD9 and TSG101, were detected in all samples using western blot analysis, thus confirming the presence of exosomes. However, the endoplasmic reticulum marker calnexin was not detected, which verified the purity of the exosomes (Fig. 1C).

DE lncRNAs and mRNAs in plasma exosomes from patients with GHGIN. To determine the expression profiles of lncRNAs in GHGIN, high-throughput RNA sequencing was used to detect the expression levels of exosomal lncRNAs and mRNAs in plasma obtained from patients with GHGIN and HC. The expression levels of IncRNAs and mRNAs in the plasma exosomes from patients with GHGIN differed 
Table III. Top 10 up- and downregulated lncRNAs and mRNAs in patients with gastric high-grade intraepithelial neoplasia compared with that in healthy controls.

\begin{tabular}{lc} 
A, Upregulated lncRNAs & \\
\hline Name & $\operatorname{logFC}$ \\
\hline RP11-290D2.6 & 1499.71 \\
XLOC_009961 & 282.17 \\
IGBP1-AS2 & 217.93 \\
XLOC_014415 & 153.39 \\
CTB-43P18.1 & 145.8 \\
RP11-223C24.1 & 105.82 \\
AC009133.15 & 95.31 \\
RP11-265N6.1 & 87.69 \\
XLOC_000702 & 84.28 \\
RP11-124N14.3 & 70.66
\end{tabular}

B, Downregulated lncRNAs

\begin{tabular}{lr}
\hline Name & $\operatorname{logFC}$ \\
\hline JA760600 & -535.73 \\
RP5-1092A3.4 & -141.12 \\
AC007228.9 & -99.88 \\
RP3-470B24.5 & -69.09 \\
RP4-790G17.7 & -67.23 \\
MIR29A & -26.01 \\
RP11-728E14.3 & -22.34 \\
\hline
\end{tabular}

C, Upregulated mRNAs

\begin{tabular}{lr}
\hline Name & \multicolumn{1}{c}{$\operatorname{logFC}$} \\
\hline BEST1 & 1041.40 \\
NRGN & 790.73 \\
FCGR2A & 244.57 \\
HLA-E & 236.44 \\
MTPN & 154.52 \\
RPL35A & 145.38 \\
BCR & 104.00 \\
WDR1 & 100.73 \\
HIST1H2BK & 95.73 \\
NUP88 & 72.43 \\
\hline
\end{tabular}

D, Downregulated mRNAs

\begin{tabular}{lr}
\hline Name & $\operatorname{logFC}$ \\
\hline MAP1LC3B & -200.72 \\
HINT3 & -104.05 \\
SCAND3 & -99.35 \\
SRRM2 & -32.84 \\
AGBL5 & -30.21 \\
PLEKHM1 & -19.42 \\
CLIP2 & -18.07
\end{tabular}

Table III. Continued.

\begin{tabular}{ll}
\hline Name & $\log \mathrm{FC}$ \\
\hline CDS2 & -17.22 \\
TNNT1 & -14.028 \\
\hline FC, fold change; lnc, long non-coding. & \\
\hline
\end{tabular}

A

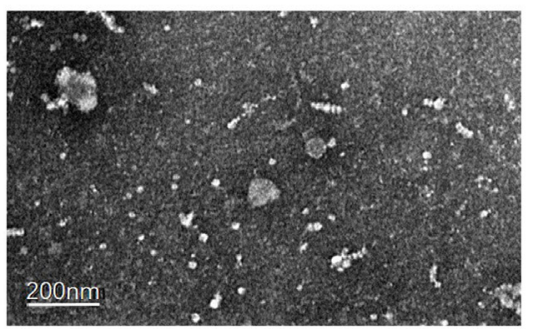

B

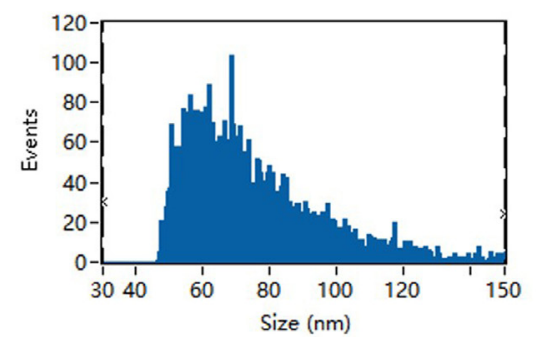

C

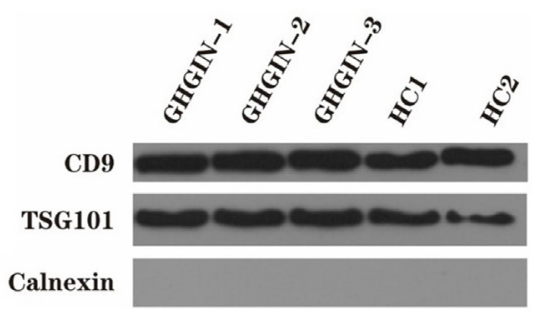

Figure 1. Exosomes from patients with GHGIN. (A) Representative TEM image of exosomes with negative staining to enhance the view of membrane structures. (B) Grain diameter distribution map of exosomes. (C) Western blot analysis to confirm pure exosomes. CD9 and TSG101 were detected in exosome samples and calnexin was used as negative control. GHGIN, gastric high-grade intraepithelial neoplasia; HC, healthy control.

significantly from that in exosomes from HC. Fig. 2A and B show the heat map depicting the expression levels of all DE lncRNAs and mRNAs, respectively. A total of 25,145 lncRNAs and 20,254 mRNAs were identified in all the samples; $83 \mathrm{DE}$ lncRNAs and 233 DE mRNAs were identified $(\log F C \geq 2.0$; $\mathrm{P} \leq 0.05)$. Among these, 76 and 7 lncRNAs were up- and downregulated, respectively. While 183 and 50 mRNAs were up- and downregulated, respectively. Table III lists the top 10 up- and downregulated lncRNAs and mRNAs.

GO and KEGG enrichment of DE lncRNAs. To identify the biological properties and functions of the DE lncRNAs, GO and KEGG pathway enrichment analyses were performed, as shown in Figs. 3A-F and 4, respectively. GO molecular function analysis indicated that upregulated DE IncRNAs were associated with 'structural constituent of myelin sheath', 
A

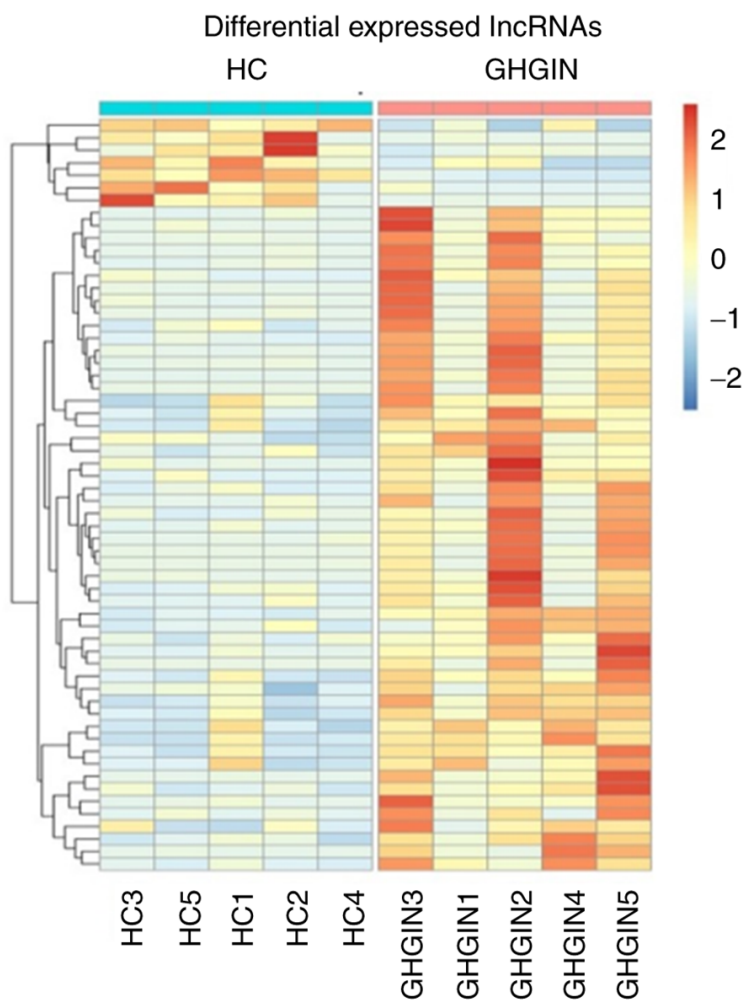

$\mathrm{B}$

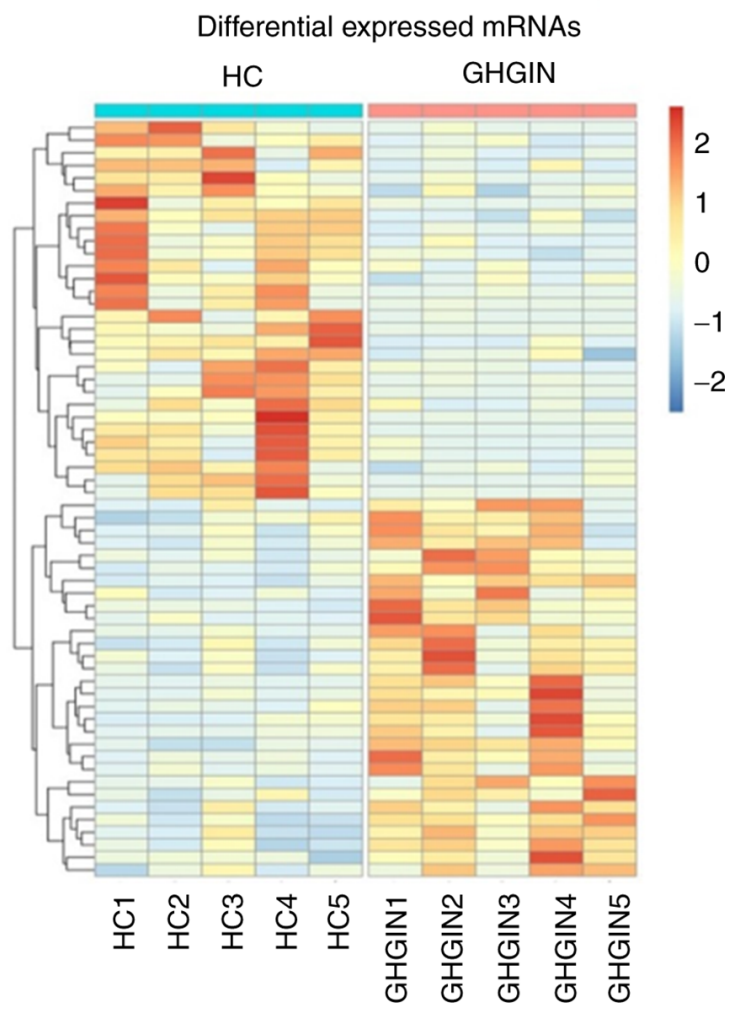

Figure 2. Cluster analysis of differentially expressed lncRNA and mRNA. Heat maps of differentially expressed (A) lncRNAs and (B) mRNAs in plasma exosomes from patients with GHGIN (red bar) compared with that in the HC (blue bar). P $<0.05$ and logFC $>2.0$. 1 nc, long non-coding; GHGIN, gastric high-grade intraepithelial neoplasia; HC, healthy controls; FC, fold-change.

'serine-type exopeptidase activity', 'catalytic activity, acting on a glycoprotein', 'voltage-gated calcium channel activity', etc. Whereas GO molecular function analysis indicated downregulated DE lncRNAs were enriched in 'ubiquitin protein ligase binding' and 'ubiquitin-like protein ligase binding'.

For GO cellular component, the upregulated DE lncRNAs were significantly enriched in 'endoplasmic reticulum', 'endoplasmic reticulum quality control compartment', 'pronucleus', 'nuclear envelope' and the extracellular space ('extracellular exosome', 'extracellular region', 'extracellular vesicle' and 'extracellular organelle'). While downregulated DE IncRNAs were enriched in 'ubiquitin ligase complex' ('SCF ubiquitin ligase complex', 'cul3-RING ubiquitin ligase complex' and 'cullin-RING ubiquitin ligase complex').

For GO biological process, upregulated DE lncRNAs were particularly involved in 'glycoprotein metabolic process', 'membrane raft localization', 'oxidative demethylation', 'histone H3-K4 trimethylation', 'transcytosis', 'energy homeostasis', 'entry of bacterium into host cell' and 'macromolecule and protein glycosylation'. Whereas downregulated DE lncRNAs were enriched in 'negative regulation of protein ubiquitination', 'negative regulation of protein modification by small protein conjugation or removal' and 'proteasomemediated ubiquitin-dependent protein catabolic process', etc.

As seen in Fig. 4, the top 10 enriched KEGG pathways were: 'Renin-angiotensin system' (RAS), 'N-glycan biosynthesis', 'arrhythmogenic right ventricular cardiomyopathy', 'cardiac muscle contraction', 'hypertrophic cardiomyopathy', 'dilated cardiomyopathy', 'Parkinson's disease', 'adrenergic signaling in cardiomyocytes', 'oxytocin signaling pathway' and the 'mitogen-activated protein kinase (MAPK) signaling pathway'.

Validation of DE lncRNA expression levels using $R T$-qPCR. RT-qPCR was performed to verify differential expression of 6 lncRNAs. A total of 5 upregulated lncRNAs (ENST00000608119, ENST00000442783, ENST00000608625, ENST00000518266 and ENST00000523150) and 1 downregulated lncRNA (ENST 00000427153) were randomly selected for validation. Significant upregulation of 5 lncRNAs was observed in samples from patients with GHGIN compared with that in the HC samples, while the expression level of ESTN00000427153 was reduced in patients with GHGIN compared with that in HC (Fig. 5), thus confirming that the lncRNA expression profile was a reliable indicator of GHGIN.

lncRNA-miRNA-mRNA interaction network. ceRNAs mutually regulate transcripts at the post-transcriptional level by competing for shared miRNAs $(36,37)$. The ceRNA network connects the function of protein-encoding mRNAs to the function of ncRNAs, such as miRNAs, IncRNAs, pseudogenic RNAs and circular RNAs (37). A lncRNA-miRNA-mRNA interaction network was constructed in accordance with the ceRNA theory, as shown in Fig. 6. A IncRNA-miRNA-mRNA interaction network with 585 edges and 178 nodes was finally obtained. A total of 7 lncRNAs, including HIF1A-AS1, NOP14-AS1, SPAG5-AS1, EAF1-AS1, IGBP1-AS2, ST7-AS2 and AC008277 were identified. In addition, 121 miRNAs and 50 mRNAs were identified. 
A

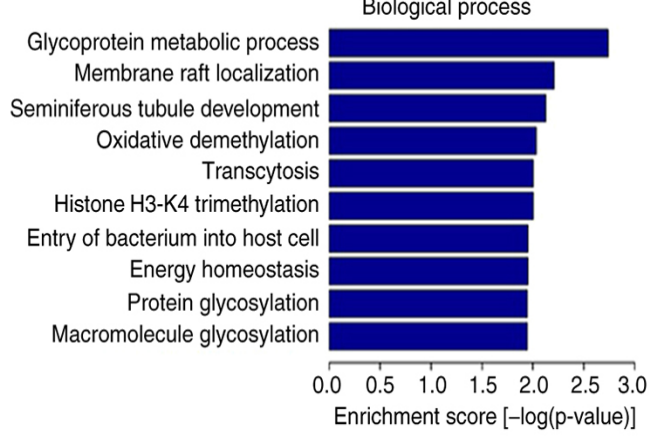

C

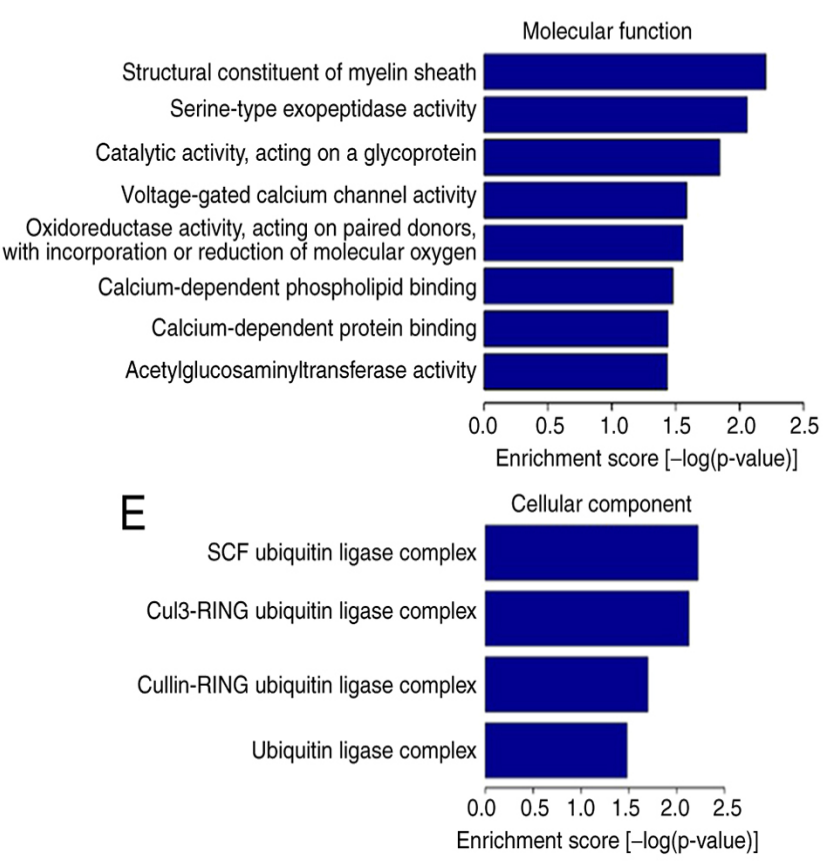

B

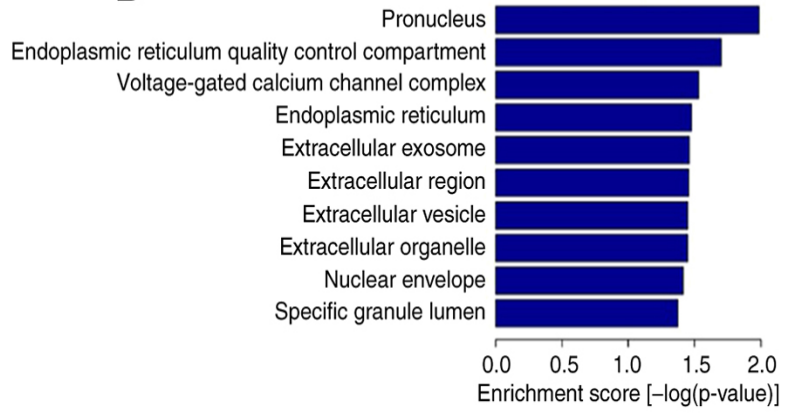

Biological process

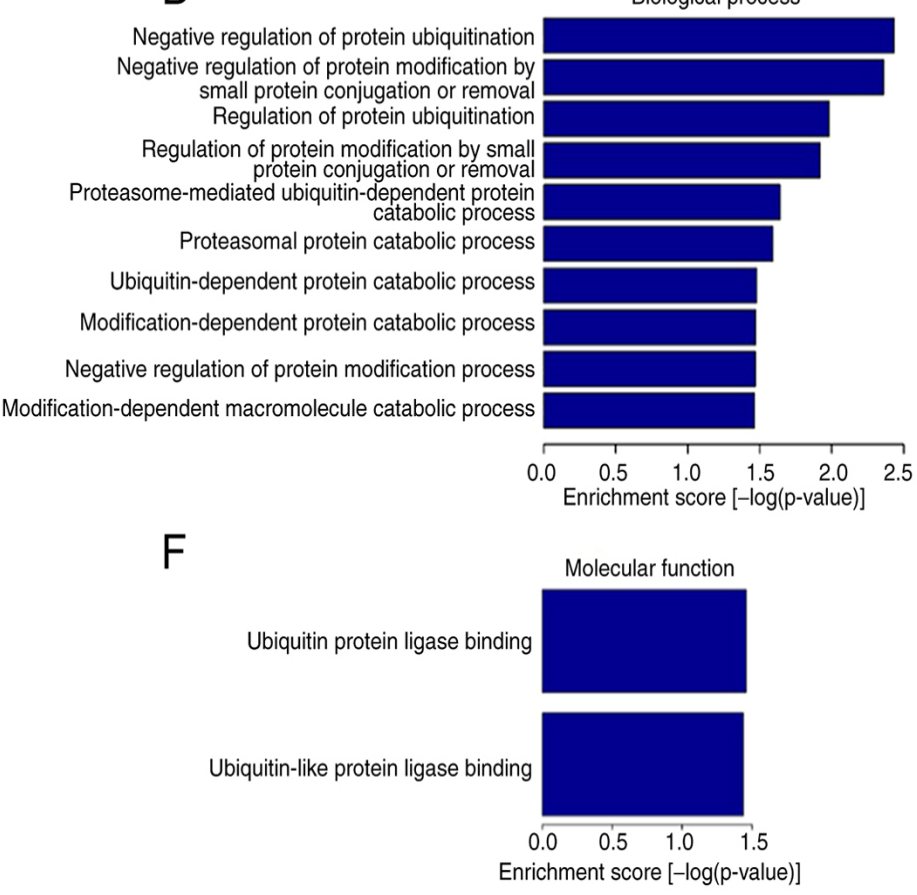

Figure 3. Top 10 differentially expressed lncRNAs GO terms. The enrichment score was calculated as -log10 (P-value). (A-C) GO analysis of upregulated lncRNAs. (D-F) GO analysis of downregulated lncRNAs. GO, Gene Ontology; lnc, long non-coding.

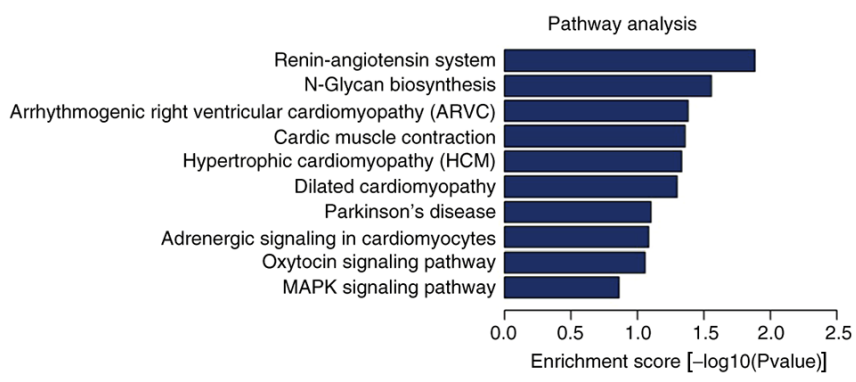

Figure 4. Kyoto Encyclopedia of Genes and Genomes pathway enrichment analysis of the top 10 upregulated differentially expressed long non-coding RNAs.

\section{Discussion}

The incidence rate of GC is declining; however, it remains one of the most common and deadly cancer types worldwide, accounting for over 700,000 deaths every year (1). Thus, early diagnosis and management of preneoplastic conditions can reduce GC-related mortality (3). Generally, GHGIN is detected using gastroscopy and biopsy, but procedure-induced discomfort causes some individuals to avoid the examination (38). In addition, detection of GHGIN requires technically skilled and experienced operators. These problems lead to a low rate of detection for GHGIN, emphasizing the requirement for non-invasive biomarkers. Elucidation of GHGIN pathogenesis and development of non-invasive, sensitive and specific biomarkers for GHGIN are required to improve the current management of GC.

Exosomes are extracellular membranous vesicles secreted by different types of cells, which are indispensable promoters of information exchange between cells. More importantly, exosomes play significant roles in multiple diseases, including cancer (13). Previous studies have demonstrated that exosomes are key molecules of cell-cell communication between cancer and stromal cells in local and distant microenvironments (39-41). IncRNAs are endogenous ncRNAs that have been associated with the occurrence, invasion and metastasis of cancer cells (15). Exosomal lncRNAs serve as messengers for intercellular communication, reshape the tumor microenvironment and have been associated with tumor proliferation, 

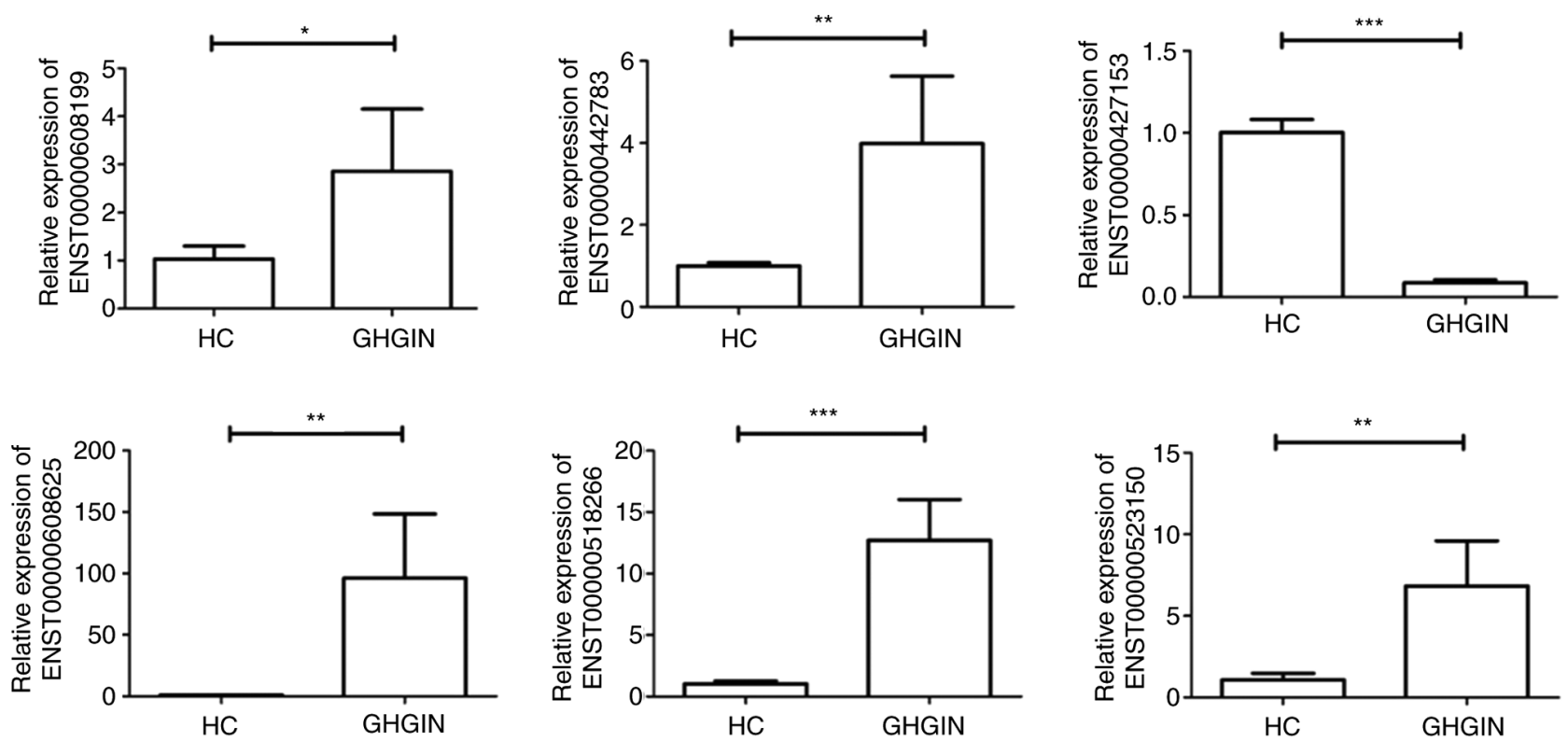

Figure 5. Reverse transcription-quantitative PCR validation of the expression levels of 6 randomly selected differentially expressed long non-coding RNAs in exosomes from patients with GHGIN and HC. ${ }^{*} \mathrm{P}<0.05,{ }^{* *} \mathrm{P}<0.01$ and ${ }^{* * *} \mathrm{P}<0.001$. HC, healthy controls; GHGIN, gastric high-grade intraepithelial neoplasia.

angiogenesis, metastasis, drug resistance and other processes, such as epithelial to mesenchymal transition and invasion (13). Gastric epithelial dysplasia is hypothesized to progress through a series of histopathological stages, from mild to severe dysplasia, to carcinoma in situ and finally to invasive GC. These histopathological grading changes are based on changes in the gross chromosome and changes in expression of protein-coding genes and ncRNAs (42).

The present study identified 83 DE lncRNAs between patients with GHGIN and HCs, including 76 upregulated and 7 downregulated lncRNAs. These DE lncRNAs, screened using RNA sequencing analysis, were further verified using RT-qPCR. GO and KEGG enrichment analyses were performed to predict the potential functions of these lncRNAs, with respect to GHGIN occurrence, and a corresponding lncRNA-miRNA-mRNA association network was constructed. According to GO analysis, the DE IncRNAs were located in the endoplasmic reticulum and extracellular space, and were associated with biological processes involved in tumorigenesis, such as protein and macromolecule glycosylation and regulation of protein ubiquitination.

Glycosylation is the most common and complex post-translational modification of cell surface and secreted proteins. Considering its critical functions and biological effects, changes in protein glycosylation are fundamental to tumorigenic transformation (43). This can decisively stimulate the progress of more malignant features, such as impaired cell-cell adhesion, enhanced migration and promotion of lymphatic metastasis (44). Aberrant glycosylation in tumor biology indicated more aggressive phenotypes in gastric cancer cell lines (45). The significant role of glycosylation in cancer is highlighted by the fact that changes in glycosylation regulate the development and progression of cancer; therefore, genes and pathways associated with glycosylation may serve as significant biomarkers, as well as specific therapeutic targets (46).
The ubiquitin-proteasome system is one of the noteworthy regulatory mechanisms that participate in protein stability (47) and cell signal transduction (48). Proteins are ubiquitinated by the synergistic effect of ubiquitin-activating enzymes. The ubiquitin-conjugating enzyme, E2O, is regularly amplified or mutated in numerous tumors, such as breast, gastric, kidney and ovarian cancer, and its high expression level was associated with low survival rates in patients with gastric, lung, breast and prostate carcinoma (49). Furthermore, the expression of ubiquitin ligase tripartite motif 59 (TRIM59) was upregulated in human gastric tumor tissues compared with that in non-tumor tissues, and its levels have been associated with cancer development and patient survival period (50). Further mechanism studies showed that the interaction between TRIM59 and P53 promoted ubiquitination and degradation, and promoted the progress of GC.

Some signaling pathways enriched in the KEGG analysis in the current study were also associated with tumor progression in GC, such as the RAS and MAPK signaling pathway. Angiotensin-converting enzyme (ACE) is the main regulatory factor of RAS and genetic polymorphisms of ACE have been associated with the risk of developing different types of human cancer, such as esophageal, gallbladder, colorectal and gastric cancer (51). RAS plays a notable role not only in physiological homeostasis but also in carcinogenesis; it is involved in numerous aspects of GC progression associated with Helicobacter pylori infection. Furthermore, RAS inhibitors reduce GC tumor development, progression and metastasis (52). Another study found that ACE and angiotensin II receptor type 2 (AT2R) were significantly upregulated in GC and metastatic tissues, while angiotensin II receptor type 1 (AT1R) expression was higher in metastatic cancer tissues compared with that found in previous investigations (53). This study found evidence of the local angiotensin II system expression in lymph node metastasis, and that ACE, AT1R and AT2R activity promoted GC cell invasion. 


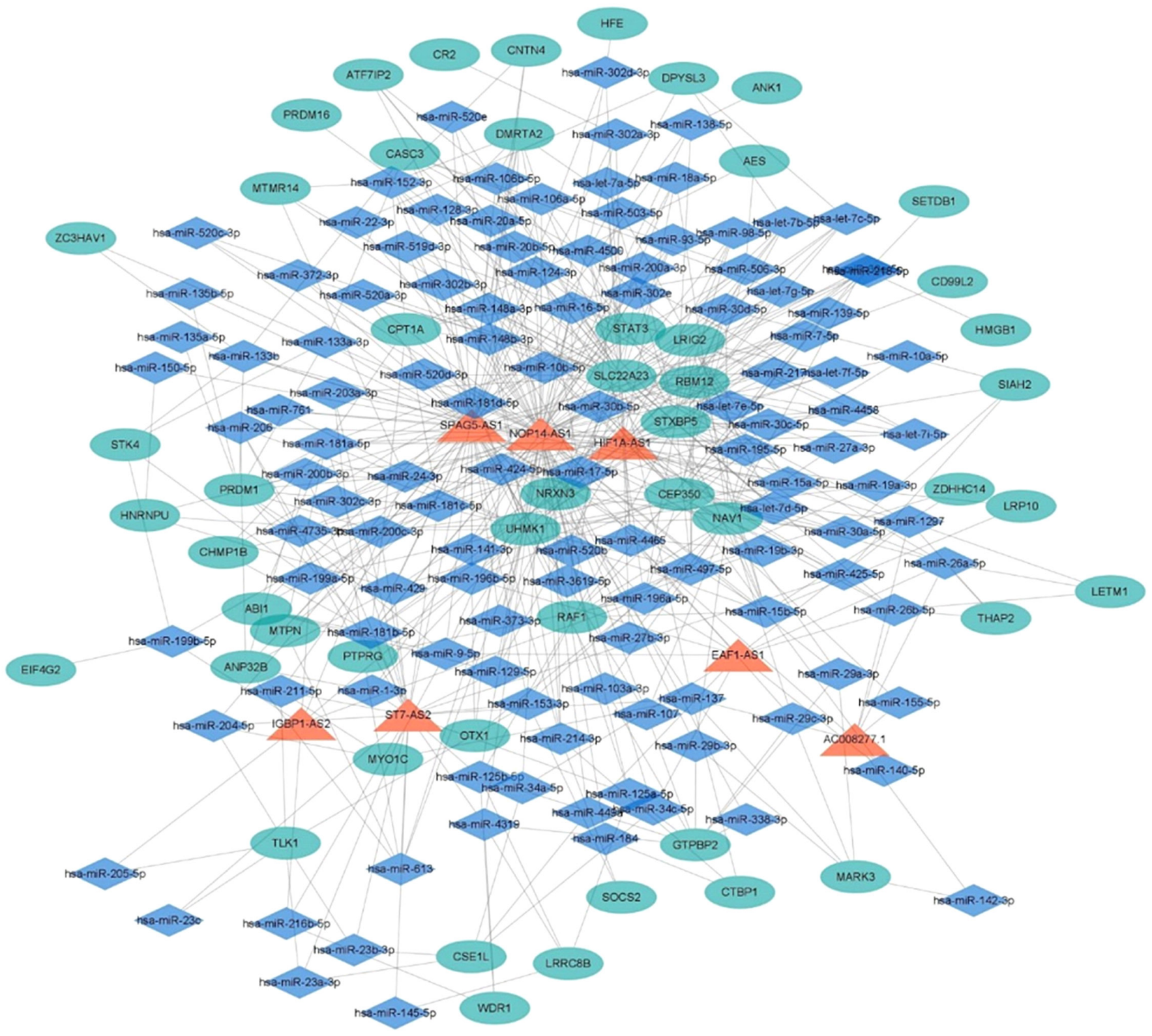

Figure 6. Construction of the IncRNA-miRNA-mRNA interaction network based on miRcode database information and high-throughput differentially expressed RNAs. Orange triangles, blue diamonds and green ellipses indicate lncRNAs, miRNAs and mRNAs, respectively. miR, microRNA; lnc, long non-coding.

The MAPK signaling pathway is widely expressed in multicellular organisms and plays a key role in various biological processes, such as cell proliferation, apoptosis, differentiation, migration and invasion (54). Yang and Huang (55) demonstated that MAPK signaling was associated with GC invasion and metastasis (55). CD97 promoted the proliferation and invasion of GC cells via the exosome-mediated MAPK signaling pathway in vitro, and exosomal miRNAs may be involved in the activation of CD97-related pathways (56). LINC00483 is an oncogenic IncRNA in GC which was found to activate the MAPK signaling pathway in GC cells and promoted GC cell proliferation, invasiveness and metastasis in vitro and in vivo (57). By regulating the key components of the aforementioned signaling pathways, IncRNAs could control GC progression.

Previous studies have demonstrated that RNAs cross-regulate each other with miRNA response elements, known as the
ceRNA hypothesis $(32,37)$. IncRNAs are suggested to play notable roles in cancer. Several studies have reported that lncRNAs interact with miRNAs and regulate their expression levels as ceRNAs (58-60). Bioinformatics analysis was used to predict whether these IncRNAs may affect the expression of differential mRNAs by high-throughput sequencing through the miRNA sponge pathway. The IncRNA-miRNA-mRNA interaction network constructed in the current study identified 7 lncRNAs, including HIF1A-AS1, NOP14-AS1, SPAG5-AS1, EAF1-AS1, IGBP1-AS2, ST7-AS2 and AC008277.1. HIF1A-AS1, an oncogene, reportedly participates in the occurrence and development of multiple types of cancer, including lung cancer, colorectal cancer and hepatocellular carcinoma (61-64). HIF1A-AS1 is elevated in a variety of cancers. In the present study, results obtained using high-throughput RNA sequencing demonstrated that HIF1A-AS1 was 
significantly increased in plasma exosomes of patients with GHIGN, prompting further study of the expression and role of this gene in GC. In addition, NOP14-AS1 was found to be upregulated in lung and liver cancer cells following exposure to DNA damage (65). Among the 50 target mRNAs shown in the interaction network, 20 were associated with the occurrence and progression of GC in PubMed. Taken together, these findings suggested that the lncRNAs and mRNAs found in GHGIN plasma exosomes were associated with the occurrence and progression of GC.

In summary, a series of exosomal DE IncRNAs were identified from the plasma of patients diagnosed with GHGIN using high-throughput RNA sequencing and their potential functions were investigated through bioinformatics analysis. The current study provides insight into the mechanism of GHGIN and aids in the development of potential biomarkers for its diagnosis, which will provide improved diagnosis and treatment of GC in the future.

\section{Acknowledgements}

The authors would like to thank Dr Huanfa Yi for invaluable advice and guidance on this manuscript for English language editing.

\section{Funding}

The present study was funded by The National Natural Science Foundation of China (grant nos. 30972610, 81273240, 91742107 and 81570002), National Key Research and Development Program (grant nos. 2017YFC0910000 and 2017YFD0501300) and Jilin Province Science and Technology Agency (grant nos. 20200403084SF, JLSWSRCZX2020-009, 20200901025SF, 20190101022JH, 2019J026, 20170622009JC, 2017C021, 2017J039, SXGJXX2017-8, JJKH20180197KJ, DBXM154-2018 and 2018SCZWSZX-015).

\section{Availability of data and materials}

The datasets generated in the present study have been uploaded to NCBI GEO (accession no. GSE153413; https://www.ncbi. nlm.nih.gov/geo/query/acc.cgi?acc=GSE153413).

\section{Authors' contributions}

FH and YJ designed the study and collected samples. MW, $\mathrm{XZ}$ and LD analyzed the data. FH, MR, MZ and QM collected data and conducted the literature review. FH and MZ drafted the manuscript. FH, MR and YJ confirm the authenticity of all the raw data. All authors have read and approved the final manuscript.

\section{Ethics approval and consent to participate}

The study protocol was approved by The Ethics Committee of the First Hospital of Jilin University, and all the procedures were performed in accordance with The Declaration of Helsinki. All participants provided written informed consent prior to the procedures.

\section{Patient consent for publication}

Not applicable.

\section{Competing interests}

The authors declare that they have no competing interests.

\section{References}

1. Bray F, Ferlay J, Soerjomataram I, Siegel RL, Torre LA and Jemal A: Global cancer statistics 2018: GLOBOCAN estimates of incidence and mortality worldwide for 36 cancers in 185 countries. CA Cancer J Clin 68: 394-424, 2018.

2. Allemani C, Weir HK, Carreira H, Harewood R, Spika D, Wang XS, Bannon F, Ahn JV, Johnson CJ, Bonaventure A, et al: Global surveillance of cancer survival 1995-2009: Analysis of individual data for $25,676,887$ patients from 279 population-based registries in 67 countries (CONCORD-2). Lancet 385: 977-1010, 2015.

3. Eusebi LH, Telese A, Marasco G, Bazzoli F and Zagari RM: Gastric cancer prevention strategies: A global perspective. J Gastroenterol Hepatol 35: 1495-1502, 2020.

4. Fassan M, Baffa R and Kiss A: Advanced precancerous lesions within the GI tract: The molecular background. Best Pract Res Clin Gastroenterol 27: 159-169, 2013.

5. Sung JK: Diagnosis and management of gastric dysplasia. Korean J Intern Med 31: 201-209, 2016.

6. Li D, Bautista MC, Jiang SF, Daryani P, Brackett M, Armstrong MA, Hung YY, Postlethwaite D and Ladabaum U: Risks and predictors of gastric adenocarcinoma in patients with gastric intestinal metaplasia and dysplasia: A population-based study. Am J Gastroenterol 111: 1104-1113, 2016.

7. Wortzel I, Dror S, Kenific CM and Lyden D: Exosome-mediated metastasis: Communication from a distance. Dev Cell 49: 347-360, 2019.

8. Meldolesi J: Exosomes and ectosomes in intercellular communication. Curr Biol 28: R435-R444, 2018.

9. Wang J, Liu Y, Sun W, Zhang Q, Gu T and Li G: Plasma exosomes as novel biomarker for the early diagnosis of gastric cancer. Cancer Biomark 21: 805-812, 2018.

10. Kahroba H, Hejazi MS and Samadi N: Exosomes: From carcinogenesis and metastasis to diagnosis and treatment of gastric cancer. Cell Mol Life Sci 76: 1747-1758, 2019.

11. Kalluri R: The biology and function of exosomes in cancer. J Clin Invest 126: 1208-1215, 2016.

12. Abak A, Abhari A and Rahimzadeh S: Exosomes in cancer: Small vesicular transporters for cancer progression and metastasis, biomarkers in cancer therapeutics. PeerJ 6: e4763, 2018.

13. Becker A, Thakur BK, Weiss JM, Kim HS, Peinado H and Lyden D: Extracellular vesicles in cancer: Cell-to-cell mediators of metastasis. Cancer Cell 30: 836-848, 2016.

14. Maia J, Caja S, Strano Moraes MC, Couto N and Costa-Silva B: Exosome-Based Cell-Cell communication in the tumor microenvironment. Front Cell Dev Biol 6: 18, 2018.

15. Wang M, Zhou L, Yu F, Zhang Y, Li P and Wang K: The functional roles of exosomal long non-coding RNAs in cancer. Cell Mol Life Sci 76: 2059-2076, 2019.

16. Kopp F and Mendell JT: Functional classification and experimental dissection of long Noncoding RNAs. Cell 172: 393-407, 2018.

17. Bhan A, Soleimani M and Mandal SS: Long Noncoding RNA and cancer: A new paradigm. Cancer Res 77: 3965-3981, 2017.

18. Jiang C, Yang Y, Yang Y, Guo L, Huang J, Liu X, Wu C and Zou J: Long Noncoding RNA (lncRNA) HOTAIR affects tumorigenesis and metastasis of non-small cell lung cancer by upregulating miR-613. Oncol Res 26: 725-734, 2018.

19. Gu L, Lu LS, Zhou DL and Liu ZC: UCA1 promotes cell proliferation and invasion of gastric cancer by targeting CREB1 sponging to miR-590-3p. Cancer Med 7: 1253-1263, 2018.

20. Sun Z, Yang S, Zhou Q, Wang G, Song J, Li Z, Zhang Z, Xu J, Xia K, Chang Y, et al: Emerging role of exosome-derived long non-coding RNAs in tumor microenvironment. Mol Cancer 17: 82, 2018.

21. Zhou R, Chen KK, Zhang J, Xiao B, Huang Z, Ju C, Sun J, Zhang F, Lv XB and Huang G: The decade of exosomal long RNA species: An emerging cancer antagonist. Mol Cancer 17: $75,2018$. 
22. Sarfi M, Abbastabar M and Khalili E: Long noncoding RNAs biomarker-based cancer assessment. J Cell Physiol 234: 16971-16986, 2019.

23. Zhao R, Zhang Y, Zhang X, Yang Y, Zheng X, Li X, Liu Y and Zhang Y: Exosomal long noncoding RNA HOTTIP as potential novel diagnostic and prognostic biomarker test for gastric cancer. Mol Cancer 17: 68, 2018.

24. Dong L, Lin W, Qi P, Xu MD, Wu X, Ni S, Huang D, Weng WW, Tan C, Sheng W, et al: Circulating long RNAs in serum extracellular vesicles: Their characterization and potential application as biomarkers for diagnosis of colorectal cancer. Cancer Epidemiol Biomarkers Prev 25: 1158-1166, 2016.

25. Li J, Peng W, Du L, Yang Q, Wang C and Mo YY: The oncogenic potentials and diagnostic significance of long non-coding RNA LINC00310 in breast cancer. J Cell Mol Med 22: 4486-4495, 2018.

26. Svoboda M, Slyskova J, Schneiderova M, Makovicky P, Bielik L, Levy M, Lipska L, Hemmelova B, Kala Z, Protivankova M, et al: HOTAIR long non-coding RNA is a negative prognostic factor not only in primary tumors, but also in the blood of colorectal cancer patients. Carcinogenesis 35: 1510-1515, 2014.

27. Bosman FT, Carneiro F, Hruban RH and Theise ND (eds): WHO Classification of Tumours of the Digestive System. 4th ed. IARC Press, Lyon, 2010.

28. Piao HY, Guo S, Wang Y and Zhang J: Exosomal long non-coding RNA CEBPA-AS1 inhibits tumor apoptosis and functions as a non-invasive biomarker for diagnosis of gastric cancer. Onco Targets Ther 13: 1365-1374, 2020.

29. The Gene Ontology Consortium: Expansion of the Gene Ontology knowledgebase and resources. Nucleic Acids Res 45 D331-D338, 2017.

30. Kanehisa M, Furumichi M, Tanabe M, Sato $Y$ and Morishima K: KEGG: New perspectives on genomes, pathways, diseases and drugs. Nucleic Acids Res 45: D353-D361, 2017.

31. Livak KJ and Schmittgen TD: Analysis of relative gene expression data using real-time quantitative PCR and the 2(-Delta Delta C(T)) method. Methods 25: 402-408, 2001

32. Tay Y, Rinn J and Pandolfi PP: The multilayered complexity of ceRNA crosstalk and competition. Nature 505: 344-352, 2014.

33. Jeggari A, Marks DS and Larsson E: MiRcode: A map of putative microRNA target sites in the long non-coding transcriptome. Bioinformatics 28: 2062-2063, 2012 .

34. Paci P, Colombo T and Farina L: Computational analysis identifies a sponge interaction network between long non-coding RNAs and messenger RNAs in human breast cancer. BMC Syst Biol 8: 83, 2014.

35. Shannon P, Markiel A, Ozier O, Baliga NS, Wang JT, Ramage D, Amin N, Schwikowski B and Ideker T: Cytoscape: A software environment for integrated models of biomolecular interaction networks. Genome Res 13: 2498-2504, 2003.

36. The research progress of ceRNA in the head and neck carcinoma Lin Chung Er Bi Yan Hou Tou Jing Wai Ke Za Zhi 32: 634-638, 2018 (In Chinese).

37. Qi X, Zhang DH, Wu N, Xiao JH, Wang X and Ma W: ceRNA in cancer: Possible functions and clinical implications. J Med Genet 52: 710-718, 2015.

38. He CZ and Zhang KH: Serum protein and genetic tumor markers of gastric carcinoma. Asian Pac J Cancer Prev 14: 3437-3442, 2013.

39. Wang S, Su X, Xu M, Xiao X, Li X, Li H, Keating A and Zhao RC: Exosomes secreted by mesenchymal stromal/stem cell-derived adipocytes promote breast cancer cell growth via activation of Hippo signaling pathway. Stem Cell Res Ther 10: 117, 2019.

40. Kahlert C, Melo SA, Protopopov A, Tang J, Seth S, Koch M, Zhang J, Weitz J, Chin L, Futreal A and Kalluri R: Identification of double-stranded genomic DNA spanning all chromosomes with mutated KRAS and p53 DNA in the serum exosomes of patients with pancreatic cancer. J Biol Chem 289: 3869-3875, 2014.

41. Wang S, Xu M, Li X, Su X, Xiao X, Keating A and Zhao RC: Exosomes released by hepatocarcinoma cells endow adipocytes with tumor-promoting properties. J Hematol Oncol 11: 82, 2018.

42. Gibb EA, Enfield KS, Stewart GL, Lonergan KM, Chari R, Ng RT, Zhang L, MacAulay CE, Rosin MP and Lam WL: Long non-coding RNAs are expressed in oral mucosa and altered in oral premalignant lesions. Oral Oncol 47: 1055-1061, 2011.

43. Stowell SR, Ju T and Cummings RD: Protein glycosylation in cancer. Annu Rev Pathol 10: 473-510, 2015.

44. Rasheduzzaman M, Kulasinghe A, Dolcetti R, Kenny L, Johnson NW, Kolarich D and Punyadeera C: Protein glycosylation in head and neck cancers: From diagnosis to treatment. Biochim Biophys Acta Rev Cancer 1874: 188422, 2020.
45. Ferreira JA, Magalhães A, Gomes J, Peixoto A, Gaiteiro C, Fernandes E, Santos LL and Reis CA: Protein glycosylation in gastric and colorectal cancers: Toward cancer detection and targeted therapeutics. Cancer Lett 387: 32-45, 2017.

46. Pinho SS and Reis CA: Glycosylation in cancer: Mechanisms and clinical implications. Nat Rev Cancer 15: 540-555, 2015.

47. Cheng J, North BJ, Zhang T, Dai X, Tao K, Guo J and Wei W: The emerging roles of protein homeostasis-governing pathways in Alzheimer's disease. Aging Cell 17: e12801, 2018.

48. Gutierrez GJ and Ronai Z: Ubiquitin and SUMO systems in the regulation of mitotic checkpoints. Trends Biochem Sci 31: 324-332, 2006.

49. Ullah K, Zubia E, Narayan M, Yang J and Xu G: Diverse roles of the E2/E3 hybrid enzyme UBE2O in the regulation of protein ubiquitination, cellular functions, and disease onset. FEBS J 286: 2018-2034, 2019.

50. Zhou Z, Ji Z, Wang Y, Li J, Cao H, Zhu HH and Gao WQ: TRIM59 is up-regulated in gastric tumors, promoting ubiquitination and degradation of p53. Gastroenterology 147: 1043-1054, 2014.

51. Abdeahad H, Avan A, Khazaei M, Soleimanpour S, Ferns GA, Fiuji H, Ryzhikov M, Bahrami A and Hassanian SM: Angiotensin-converting enzyme gene polymorphism and digestive system cancer risk: A meta-analysis based on 9656 subjects. J Cell Biochem 120: 19388-19395, 2019.

52. Sugimoto M, Yamaoka Y, Shirai N and Furuta T: Role of renin-angiotensin system in gastric oncogenesis. J Gastroenterol Hepatol 27: 442-451, 2012.

53. Carl-McGrath S, Ebert MP, Lendeckel U and Röcken C: Expression of the local angiotensin II system in gastric cancer may facilitate lymphatic invasion and nodal spread. Cancer Biol Ther 6: 1218-1226, 2007.

54. Yang SH, Sharrocks AD and Whitmarsh AJ: MAP kinase signalling cascades and transcriptional regulation. Gene 513: 1-13, 2013.

55. Yang $M$ and Huang CZ: Mitogen-activated protein kinase signaling pathway and invasion and metastasis of gastric cancer. World J Gastroenterol 21: 11673-11679, 2015.

56. Li C, Liu DR, Li GG, Wang HH, Li XW, Zhang W, Wu YL and Chen L: CD97 promotes gastric cancer cell proliferation and invasion through exosome-mediated MAPK signaling pathway. World J Gastroenterol 21: 6215-6228, 2015.

57. Li D, Yang M, Liao A, Zeng B, Liu D, Yao Y, Hu G, Chen X, Feng Z, Du Y, et al: Linc00483 as ceRNA regulates proliferation and apoptosis through activating MAPKs in gastric cancer. J Cell Mol Med 22: 3875-3886, 2018.

58. Guo LL, Song CH, Wang P, Dai LP, Zhang JY and Wang KJ: Competing endogenous RNA networks and gastric cancer. World J Gastroenterol 21: 11680-11687, 2015.

59. Tay Y, Kats L, Salmena L, Weiss D, Tan SM, Ala U, Karreth F, Poliseno L, Provero P, Di Cunto F, et al: Coding-independent regulation of the tumor suppressor PTEN by competing endogenous mRNAs. Cell 147: 344-357, 2011.

60. Huarte M: The emerging role of lncRNAs in cancer. Nat Med 21: 1253-1261, 2015

61. Wu Y, Liu H, Shi X, Yao Y, Yang W and Song Y: The long non-coding RNA HNF1A-AS1 regulates proliferation and metastasis in lung adenocarcinoma. Oncotarget 6: 9160-9172, 2015.

62. Zhu W, Zhuang P, Song W, Duan S, Xu Q, Peng M and Zhou J: Knockdown of lncRNA HNF1A-AS1 inhibits oncogenic phenotypes in colorectal carcinoma. Mol Med Rep 16: 4694-4700, 2017.

63. Gao J, Cao R and Mu H: Long non-coding RNA UCA1 may be a novel diagnostic and predictive biomarker in plasma for early gastric cancer. Int J Clin Exp Pathol 8: 12936-12942, 2015.

64. Hong F, Gao Y, Li Y, Zheng L, Xu F and Li X: Inhibition of HIF1A-AS1 promoted starvation-induced hepatocellular carcinoma cell apoptosis by reducing HIF-1 $\alpha /$ mTOR-mediated autophagy. World J Surg Oncol 18: 113, 2020.

65. Goyal A, Fiškin E, Gutschner T, Polycarpou-Schwarz M, Groß M, Neugebauer J, Gandhi M, Caudron-Herger M, Benes V and Diederichs S: A cautionary tale of sense-antisense gene pairs: Independent regulation despite inverse correlation of expression. Nucleic Acids Res 45: 12496-12508, 2017.

This work is licensed under a Creative Commons Attribution-NonCommercial-NoDerivatives 4.0 International (CC BY-NC-ND 4.0) License. 